\title{
Medical emergency announcements on cruise ships: an audit of outcome
}

\author{
Christopher James Taylor
}

Cruise Ship Physician, Nottinghamshire, England

\begin{abstract}
Background: Public address announcements are an effective way of alerting staff on cruise ships to life -threatening medical emergencies on-board, but should only be used when truly necessary. An audit to investigate the outcome following this method of activating the medical emergency response team (MERT) suggested system flaws. A new elementary first aid training programme for the crew was then developed, emphasising patient assessment and the correct determination of appropriate levels of response. Following fleet-wide implementation, post-intervention audits were performed on two other company ships to evaluate the impact of the new approach.

Materials and methods: Data from all MERT activations initiated by public address announcement were prospectively collected during the audit periods, including subsequent means of transfer to the ship's medical centre and duration of medical intervention as indicators of clinical severity.

Results: After changing the training programme the overall rate of public announcements for medical emergencies fell by $43 \%$. The proportion of patients requiring transfer by stretcher increased from $5 \%$ to $33 \%$, whilst the proportion of patients requiring $\geq 4 \mathrm{~h}$ of medical intervention increased from $5 \%$ to $44 \%$. Conclusions: The audits suggest that the new training programme may have improved the first aid responders' decision-making as there were fewer inappropriate emergency announcements over the public address system. However, two-thirds of all MERT activations were still for patients either well enough to walk or only needing a wheelchair for subsequent transfer, indicating ongoing opportunity for improvement.

(Int Marit Health 2015; 66, 4: 203-206)

\section{Key words: seafarer, first aid training, education, medical emergency response team, code, passenger} ship, STCW 1978
\end{abstract}

\section{INTRODUCTION}

'STCW 1978' are Standards of Training, Certification and Watchkeeping for seafarers set by the International Maritime Organisation and include elementary first aid training for all seafarers [1]. The purpose of this mandatory course is to enable all seafarers to take immediate and reasonable action upon encountering an accident or other medical emergency.

On cruise ships, "raising the alarm" equates to notifying the on duty medical officer(s). This usually involves a telephone call to the ship's bridge via the on-board emergency number. If the bridge officer either suspects or cannot exclude a life-threatening condition, such as heart attack, unconsciousness or heavy bleeding, a pre-designated 'code' announcement is made over the ship's public address system to activate the medical emergency response team (MERT). The MERT, comprised of all on-board medical officers, stretcher team members, on-duty security officers and a selection of senior ship management, will then respond immediately to the scene.

Whilst working as a cruise ship's doctor, the author sensed that many of the medical emergency codes broadcast over the public address system were unnecessary, since most patients had relatively minor medical complaints.

An audit conducted during a later work contract confirmed that activation of the MERT on many occasions was not in accordance with STCW regulations (see Results) [1]. 
Reasoning that training was an area for improvement, the author created a first aid training video for the crew, focusing on the initial 'lay-person' assessment of a casualty and how to call for an appropriate level of medical assistance. In 2013 it was distributed to all ships in the company's fleet and became the basis for a new teaching programme in elementary first aid.

To review the impact of this intervention, the author carried out follow-up audits of medical emergency code calls in 2014/2015, when working on two other cruise ships operated by the same company. This report presents and discusses the findings of the initial and the follow-up audits.

\section{MATERIALS AND METHODS}

The responsibility for training crew-members in elementary first aid and how to respond when faced with emergency clinical situations, in accordance with STCW regulations [1], lies with the ship's medical staff. At the time of the initial audit, medical staff had access to a computer slide presentation on which to base their teaching, although, in the author's experience, the extent to which this was used varied greatly from one practitioner to the next.

The initial, pre-intervention audit was conducted on a large cruise ship (Ship 1; Table 1) during a period of 15 weeks (24 April - 07 August 2010). This, as with the follow-up audits, involved recording anonymous information following every activation of the ship's MERT by code call throughout the author's working contract on that ship. Two factors were recorded as indicators of clinical severity: the means of patient transportation from the scene (stretcher, wheelchair or walking) and whether the patient's subsequent stay in the ship's medical centre was more or less than $4 \mathrm{~h}$.

After reviewing the findings and identifying crew training as an area for improvement (see Results), the author created a first aid training video for the crew, focusing on understanding the difference between life-threatening cases requiring the immediate response of the entire MERT and less urgent cases where notification of the duty medical officer (nurse) alone is more appropriate. After approval by the flag state (Bahamas Maritime Authority) in 2013,

Table 1. Populations audited before (Ship 1) and after (Ships 2 and 3) fleet-wide implementation of a new mandatory first aid training programme for cruise ship crew-members

\begin{tabular}{llll}
\hline & Ship 1 & Ship 2 & Ship 3 \\
\hline $\begin{array}{l}\text { Median shipboard } \\
\text { population (persons) }\end{array}$ & 7870 & 5374 & 4392 \\
$\begin{array}{l}\text { Duration of audit (days) } \\
\begin{array}{l}\text { Audit sample size } \\
\text { (persons } \times \text { days) }\end{array}\end{array}$ & 105 & 70 & 63 \\
\hline
\end{tabular}

the video was distributed fleet-wide and became the basis for the mandatory first aid training programme for all crew-members.

To evaluate the impact of this intervention, two follow-up audits were conducted; each was on a ship with a smaller onboard population (Table 1) and, being limited by the author's lengths of contract, was for a shorter duration than the initial pre-intervention audit: 10 weeks (Ship 2: 31 May - 09 August 2014) and 9 weeks (Ship 3: 07 December 2014 - 07 February 2015), respectively. The data from the latter two audits were combined to better approximate the size of the initial study.

\section{DATA CALCULATIONS}

Audit sample size was calculated as the median onboard population during the audit period multiplied by the number of days surveyed. The incidence of MERT code activation was calculated by dividing the number of activations by the sample size.

\section{ETHICS AND STATISTICS}

The primary aim of the audits was to evaluate 'standard of care' and the data were registered anonymously without further patient information. Accordingly, ethics committee approval was not required [2]. No statistical calculations were attempted.

\section{RESULTS}

Table 1 shows each of the shipboard populations involved in the audits. Table 2 shows the breakdown of outcomes following each case of activation of the MERT.

During the 15-week period of the initial audit, a total of 20 MERT activation calls were made over the public address system. The ship's median onboard population was 7,870 persons. The subsequent audits covered episodes of 10 weeks and 9 weeks, with median onboard populations of 5,374 and 4,392 persons, respectively.

The sample size of the initial audit was 826,350 person-days. The combined size of the follow up audits was $652,876(376,180+276,696)$ person-days.

The incidence of MERT activations by means of public address system in the initial audit was $2.42 \times 10^{-5}$ ( 20 cases divided by 826,350 person-days). The corresponding figure for the combined follow up audits was $1.38 \times 10^{-5}$ ( 9 cases divided by 652,876 person-days). This represents a $43 \%$ reduction in the frequency of medical emergency calls being made over the public address system.

In the initial audit there was no case of cardiac arrest. In the combined follow-up audits there were 3 cardiac arrest cases. These cases were transported to the medical centre by stretcher and categorised as requiring treatment and observation time of at least $4 \mathrm{~h}$, regardless of resus- 
Table 2. Medical Emergency Response Team activations by public announcement and indicators of case severity before (Ship 1) and after (Ships 2 and 3) fleet-wide implementation of a new mandatory first aid training programme for cruise ship crew-members

\begin{tabular}{lllll}
\hline & Ship 1 & Ship 2 & Ship 3 & Ship 2 + Ship 3 \\
\hline Emergency codes called & 20 & 5 & 4 & 9 \\
Transferred by stretcher & $1(5 \%)$ & 2 & 1 & $3(33 \%)$ \\
Transferred by wheelchair & $15(75 \%)$ & 2 & 0 & $5(56 \%)$ \\
Transferred by walking & $2(10 \%)$ & 1 & 0 & $1(11 \%)$ \\
Did not transfer to the medical centre & $2(10 \%)$ & 0 & 2 & $0(0 \%)$ \\
Treatment and observation of at least 4 $\mathrm{h}$ & $1(5 \%)$ & 2 & $4(44 \%)$
\end{tabular}

citation outcome and treatment duration, to reflect their clinical severity.

Including the cardiac arrest cases, the proportion of high severity patients requiring at least $4 \mathrm{~h}$ of medical intervention increased in the second audit, from $5 \%$ to $44 \%$, compared to the initial audit.

\section{DISCUSSION}

Proper and timely assessment, communication and coordination of response are crucial components when dealing with all kinds of emergencies, and particularly life-threatening medical emergencies at sea. STCW requires that the priority and sequence of action should be "proportional to any potential threats to life" and that "the manner and timing of raising the alarm is appropriate to the circumstances" [1]. Inappropriate raising of alarms involving the ship-wide public address system can have significant adverse impact not only on passenger leisure activities but also on crew members' statutory resting periods [3]. Each activation of the MERT requires individuals to run immediately to the scene; a potentially hazardous undertaking on a moving vessel at sea. On-going passenger service and patient care may also have to be temporarily suspended until the nature of the new emergency is known. Thus an onboard culture with an inappropriately low threshold for activating the MERT, in circumstances where a straightforward call to the nurse on duty would suffice, can have significant implications.

The majority of immediately life-threatening medical conditions might reasonably be expected to involve a patient insufficiently well for sitting upright or walking. In the initial audit only 1 (5\%) of 20 cases in which the MERT was activated resulted in transfer to the ship's medical centre by stretcher, indicating that the governing STCW principles were not being closely followed [1].

Subsequent to the findings of the initial audit, the new training programme for crew-members was developed with a specific focus on assessing a casualty and determining appropriate response levels before calling for assistance. An instructional video was produced covering all aspects of the elementary first aid training as mandated by STCW [1]. It emphasised that the ship's bridge should only be called on the emergency number to report potentially life-threatening conditions; all other medical emergencies should be reported by calling the nurse on duty directly, who can then attend the scene and determine the level of required response. No changes were made to the bridge officers' protocols.

The combined post-intervention audits showed a 43\% fall in MERT activations, accompanied by a greater proportion of patients requiring stretcher transport and prolonged medical care. These findings suggest improved judgment by first responders when activating the MERT.

The simple criteria for classifying the emergency as serious (needing stretcher transport to the medical centre, involving more than $4 \mathrm{~h}$ of treatment or observation) are intuitive rather than evidence-based. It is not suggested that transfer by stretcher equates to a serious life-threatening condition; dislocated joints and lower limb fractures, for instance, are not immediately life-threatening but transfer by stretcher is appropriate. However, the converse is usually true: most immediately life-threatening conditions requiring an on-scene MERT presence will require transfer by stretcher.

One confounding factor is that on this cruise line any patient requiring stretcher transport will invariably trigger activation of the MERT by public announcement because currently there is no other way to speedily gather a stretcher-carrying team. Whilst possibly affecting the absolute number of announcements, this policy remained constant across both studies so would be expected to affect both counts similarly. Furthermore, this feature counters the possibility that the new training programme resulted in instances of failure to activate the MERT when it was truly needed, since data from all patients sufficiently unwell to require stretcher transfer would automatically be captured.

It should be noted that the audits do not say anything about how many real emergencies there were on the 3 ships during the audit periods; they only registered how often the MERT was activated. They do not give any information about the numbers of emergency calls that the bridge officer sim- 
ply transferred to the nurse or how many emergency calls were directly reported to the phone number of the nurse on duty and handled without involving the response team. However, the purpose of public address system announcements is to rapidly mobilize a large team to the scene of a life-threatening medical emergency and it was the response to these scenarios that was being investigated.

In contrast to research, which aims to derive generalisable new knowledge through studies that generate hypotheses and studies that test them, an audit is a quality improvement process that seeks to answer the question: "Does this service reach a predetermined standard?" [2].

The audits presented here have their limitations. They did not register whether the caller was a passenger, a crew-member or the nurse on duty. Use of the official emergency number by passengers would not have been influenced by the new crew training course, but it is likely that the mandatory new first aid course has educated the crew-members who report emergencies and also the bridge officers who activate the emergency code, and thus may have contributed to the positive results shown by the follow-up audits.

The follow-up audits were carried out for shorter periods of time and on 2 ships, both also large but smaller in size than the original. Although the same new first aid programme had been implemented on all the ships of the fleet, and the crew-members received standardised and not ship-specific training in elementary first aid, it could be that concern regarding a perception of longer response times on the largest ship influenced the judgment of first aiders.

Other - unknown - factors may also have interfered, and despite the fact that the audits covered a high number of person-days on board, the number of medical emergency code announcements, both during the initial and the follow-up audits, were so small that significance statistics are not relevant, and chance may well have influenced the results.

Interestingly, the presented audits show that on even the largest cruise ships activation of the medical emergency code announcement is not common, yet many - if not most - of them could be handled in a less dramatic and less intrusive manner. "Better safe than sorry" is a particularly important principle on ships and when in doubt a code should be called, but for reasons already described, every unnecessary call that can be avoided is a valuable contribution and mirrors STCW objectives [1].

Even after implementation of the new crew-member first aid training programme, in two-thirds of cases a public announcement was made for a patient who subsequently did not require a stretcher transfer to the ship's medical centre. If this truly reflects optimised crew member decision making in emergency medical situations, then it might be reasonable for all emergency calls to be received directly by the nurse on duty who, after immediately responding to the scene, would then determine whether a public address announcement to summon extra assistance is required or not. Such an approach might be anticipated to further reduce by two-thirds the number of public address system announcements being made, and, since the combined data from all audits in this report reveal a cardiac arrest incidence of 3 in 1,479,226 person-days $(826,350+652,876)$, the likelihood of delaying MERT arrival for a cardiac arrest situation would appear to be very small indeed.

Whilst there is a natural tendency towards frustration from responders in the face of medically unwarranted calls such calls can still be regarded as good opportunities to practice emergency team work; followed by a proper debriefing they are more realistic and can therefore be more suitable code drills than those arranged routinely for compliance with statutory obligations.

\section{CONCLUSIONS}

In conclusion, even limited audits can uncover important information to reduce the use of unnecessary procedures while improving safety, and they may provide valuable input for medical research projects. The findings of the present audits suggest that the restructuring of crew-member first aid education, with the intent of reducing inappropriate calls, has been successful, and this has been accomplished without compromising calls necessary for the saving of life or limb. However, with two-thirds of all MERT activations still for patients who are either well enough to walk or only need a wheelchair for subsequent transfer, there remains significant room for further improvement.

\section{REFERENCES}

1. International Maritime Organization. The Manila Amendments to the Seafarers' Training, Certification and Watchkeeping (STCW) Code. STCW/CONF.2/34. 3 August 2010. http://www.imo.org/en/ KnowledgeCentre/InformationResourcesOnCurrentTopics/InformationResourcesOnCurrentTopicsArchives/Documents/STCW.pdf (accessed $2^{\text {nd }}$ October, 2015).

2. Health Research Authority. Defining Research. NRES guidance to help you decide if your project requires review by a Research Ethics Committee. Leaftet \#0987. December 2009 (rev. April 2013). http://www.hra.nhs.uk/documents/2013/09/defining-research. pdf (accessed $2^{\text {nd }}$ October, 2015).

3. International Labour Conference. Maritime Labour Convention, 2006. http://www.ilo.org/wcmsp5/groups/public/--ed_norm/-normes/documents/normativeinstrument/wcms_090250.pdf (accessed $2^{\text {nd }}$ October, 2015). 